\title{
Impact of head immobilization position on dose distribution in patients of brainstem glioma
}

\author{
Seema Sharma, Pritee Chaudhari, Ahitagni Biswas, Durai Manigandan, Peeyush Shukla, \\ Vellaiyan Subramani, Subhash Chander, Pramod Kumar Julka, Goura Kishor Rath
}

Department of Radiation Oncology, All India Institute of Medical Sciences, New Delhi, India

Received September 25, 2014; Revised January 05, 2015; Accepted January 07, 2015; Published Online January 17, 2015

\section{Technical Report}

\begin{abstract}
Purpose: The purpose of this study is to investigate the impact of patient position (supine and prone) on conventional bilateral field, three dimensional conformal radiotherapy (3DCRT) and intensity modulated radiotherapy (IMRT) treatment plans in patients of brainstem glioma with a view to exploring the possibility of avoiding beam entry through immobilization accessories. Methods: Five patients of brainstem glioma were immobilized and scanned in supine and prone positions with a combination of head rest and thermoplastic cast. Each patient was planned with three techniques: (i) 2-fields bilateral (ii) 3-fields 3DCRT, and (iii) 5-fields IMRT. Plan quality was analyzed in terms of planning target volume (PTV) coverage and dose to various critical organs at risk (OAR) for both the supine and prone treatment positions. Results: In case of bilateral fields (parallel opposed) planning, the PTV coverage and dose to the OAR were almost similar for both the supine and prone positions. In 3DCRT plan, although the PTV coverage and dose to critical structures were comparable for both the supine and prone position, dose to cochlea was lower for the prone position plan. A modest decrease in maximum dose to optic nerves and mean dose to temporal lobes were also observed for the prone position plan. In IMRT plans, the PTV coverage and homogeneity were comparable in both the supine and prone positions. Reduction in average maximum and mean doses to all OARs with functional subunit (FSU) in series and parallel respectively was observed in the IMRT plan for prone position when compared to the supine position. Conclusion: Supine and prone positions resulted in almost similar dose distribution in all the three techniques applied. At some instances, the prone position showed better normal tissues sparing when compared to supine. Moreover, prone position is more likely to avoid attenuation due to immobilization devices and uncertainty in dose calculation under large inhomogeneities.
\end{abstract}

Keywords: Brainstem Glioma; Immobilization Position; Dose distribution; Calculation Algorithm

\section{Introduction}

The immobilization accessories used for patient positioning are not completely radio-translucent and sometime large air gap may be created in the path of the beam before entering the patient body. This may result in uncertainty in dose calculation in absence of proper dose calculation algorithm. ${ }^{1}$ American association of physicists in medicine (AAPM) Task Group 176 also illustrates the dosimetric effects of a wide range of devices used for immobilization of the patient.

Complex combination of devices used external to the patient may result in increased skin dose, reduced tumor dose, and altered dose distribution. Although most treatment planning systems are capable of inclusion of the treatment couch top into the planning computed tomography (CT) and dose calculation, it may be prudent to avoid beam-entry through the more attenuating sections of the treatment couch by judicious choice of gantry angle. ${ }^{2}$ With a view to exploring the possi- bility of avoiding beam entry through immobilization accessories, we conducted a dosimetric study to analyze the impact of patient position (supine and prone) on conventional bilateral field, three dimensional conformal radiotherapy (3D-CRT) and intensity modulated radiotherapy (IMRT) treatment plans in patients of brainstem glioma. Plan quality was analyzed in terms of target coverage, dose homogeneity, and critical organ sparing in both the afore-mentioned treatment positions.

\section{Methods and Materials}

Five patients of brainstem glioma were immobilized in two ways using supine and prone position head rest with thermoplastic cast (Uniframe head mask, Med-Tec ${ }^{\mathrm{TM}}$, Orange city, IA, USA). The CT simulation with intravenous contrast

Corresponding author: Seema Sharma; Department of Radiation Oncology, All India Institute of Medical Sciences, New Delhi, India. 
was done using Philips Brilliance CT (Philips medical system, Cleveland, USA) at $3 \mathrm{~mm}$ slice thickness. The CT dataset was transferred to the Eclipse treatment planning system (TPS), version: 6.5 (Varian Medical Systems, Palo Alto, CA, USA). Dose-limiting organs at risk (OAR), i.e., optic chiasma, bilateral eye, optic nerve, temporal lobe, cochlea, and parotid were delineated on both CT sets. Gross tumor volume (GTV) was defined as tumor visible on contrast enhanced planning $\mathrm{CT}$ and diagnostic magnetic resonance (MR) images. An isotropic expansion of $1 \mathrm{~cm}$ was used over GTV to define the clinical target volume (CTV). A further $5 \mathrm{~mm}$ margin was added to CTV to form the planning target volume (PTV). Target delineation in this cohort of 5 patients was done and verified uniformly by 2 radiation oncologists in both the treatment positions. Subsequently radiation planning was done and verified uniformly by 2 medical physicists in both treatment positions.

Each patient was planned in three ways in Eclipse TPS: (i) 2-field conventional-bilateral (ii) 3-field-3DCRT and (iii) 5-field- IMRT. The prescription dose was $56 \mathrm{~Gy}$ in 28 fractions over 5.5 weeks. Treatment was delivered on Varian CL2300C/D linear accelerator having 40 pairs of multi-leaf collimator (MLC) with $1 \mathrm{~cm}$ leaf thickness at isocenter.

For bilateral 2-field planning, left lateral and right lateral field portals with $6 \mathrm{MV}$ X-rays were used, and the PTV was shaped using MLC. Field weight was kept 1:1 for left and right lateral fields, and the prescription isodose was $95 \%$.

For supine position in 3DCRT plan, two lateral oblique fields (left posterior oblique gantry angle- $110^{\circ}$, right posterior oblique gantry angle- $248^{\circ}$, and beam energy-6MV X-rays) with wedge and one superior vertex field (gantry angle- $310^{\circ}$, couch angle- $90^{\circ}$, wedge $30^{\circ}-45^{\circ}$, and beam energy- $15 \mathrm{MV}$ $\mathrm{X}$-rays) were used. Similarly for prone position in 3DCRT plan, two lateral oblique fields (left posterior oblique gantry angle- $298^{\circ}$, right posterior oblique gantry angle- $68^{\circ}$, and beam energy-6MV X-rays) with wedge and one posterior vertex field (gantry angle- $318^{\circ}$, couch angle- $270^{\circ}$, wedge $30^{\circ}-45^{\circ}$, and beam energy-15MV X-rays) were used. Plan normalization was done at isocenter and prescription isodose was $95 \%$.

For IMRT, five $6 \mathrm{MV} \mathrm{X}$-rays coplanar fields with gantry angle $0^{0}, 52^{\circ}, 104^{\circ}, 260^{\circ}$, and $312^{0}$ were used in both supine and prone positions. IMRT planning in patients of brain stem glioma was done with five equally spaced coplanar beams keeping in mind the small central nature of the target volume. All the input dose constraints in IMRT plan were achieved by above mentioned field arrangement. The prescription isodose was 95\%. Dynamic IMRT was planned using Varian Helios dose optimization using following dose volume constraints:
PTV-95\% volume should get $\geq 95 \%$ of the prescription dose, right and left eye maximum dose $\leq 45 \mathrm{~Gy}$, right and left optic nerve maximum dose $\leq 54 \mathrm{~Gy}$, optic chiasma maximum dose $\leq$ $54 \mathrm{~Gy}$, right and left temporal lobe maximum dose $\leq 60 \mathrm{~Gy}$, right and left cochlea maximum dose $\leq 54 \mathrm{~Gy}$, right and left parotid gland mean dose $\leq 26 \mathrm{~Gy}$. Plan was optimized to obtain the maximum possible sparing of OAR without compromising the PTV coverage.

For all plans, pencil beam dose calculation algorithm (version 6.5) and equivalent tissue air method were used for inhomogeneity correction at $2.5 \mathrm{~mm}$ grid size. Plan evaluation was done using dose volume histogram (DVH) in terms of target coverage, dosimetric heterogeneity and maximum and mean doses to target. For the target volume, coverage index and heterogeneity index were calculated using following relations. $^{3}$

\section{Coverage index (CI)}

$\mathrm{CI}$ is defined as the ratio of TVRI to TV

$$
\mathrm{CI}=\mathrm{TV} \mathrm{RI} / \mathrm{TV}
$$

where, $\mathrm{TV}_{\mathrm{RI}}=$ target volume covered by the reference isodose and $\mathrm{TV}=$ target volume.

\section{Heterogeneity index (HI)}

$\mathrm{HI}$ is defined as the ratio of $\mathrm{D}_{5 \%}$ to $\mathrm{D}_{95} \%$ for the PTV.

$$
\mathrm{HI}=\mathrm{D} 5 \% / \mathrm{D}_{95 \%}
$$

Where, D5\% and D95\% correspond to the dose delivered to 5\% and $95 \%$ of PTV volume respectively. For critical structures, maximum and mean doses were noted using DVH.

\section{Results}

The results are summarized in Table 1 . Figure 1 shows the typical beam arrangements and dose distribution of a representative patient for (a) 2-F bilateral in supine position (b) 2-F bilateral in prone position (c) 3D-CRT in supine position (d) 3D-CRT in prone position (e) IMRT in supine position (f) IMRT in prone position.

\section{PTV}

The maximum and minimum value of CI were observed as $0.9824 \pm 0.01$ (IMRT supine) and $0.9525 \pm 0.03$ (2F-bilateral supine), respectively. The maximum and minimum $\mathrm{HI}$ value were observed as $1.0896 \pm 0.02$ (3F-3DCRT supine) and $1.0817 \pm 0.02$ (2F-bilateral supine), respectively. Among three planning methods applied in the present study, for both the supine and prone positions, 3F-3DCRT supine position showed the lowest PTV Dmax (6077.58 \pm 91.61 cGy), whereas the highest PTV Dmax $(6327.16 \pm 66.00 \mathrm{cGy})$ was observed in supine position IMRT plan.

The maximum and minimum mean dose to PTV were $5960.62 \pm 44.86 \mathrm{cGy}$ (supine position IMRT, which was $6.43 \%$ higher compared to prescription dose) and 5855.26 \pm 45.39 
cGy (3F-3DCRT prone position, which was $4.55 \%$ higher to prescription dose), respectively.

\section{OARs}

The highest and lowest maximum dose received by optic chiasma were $5455.95 \pm 228.67$ cGy (3F-3DCRT prone) and $4861.63 \pm 507.22$ cGy (IMRT prone), respectively. The highest and lowest maximum dose to right and left optic nerve were $2402.12 \pm 784.89$ cGy (3F-3DCRT supine), $237.08 \pm$ 148.90 cGy (2F-bilateral prone) and $2151.96 \pm 933.12$ cGy (3F-3DCRT supine), $300.88 \pm 230.04$ cGy (2F-bilateral prone), respectively. The maximum dose to right and left eye were (observed in IMRT supine position plan) $1441.38 \pm 818.03$ cGy and $1211.1 \pm 717.47$ cGy, respectively. The highest and lowest mean dose to right and left cochlea were 5867.74 \pm 164.28 cGy (2F-bilateral prone), $5882.24 \pm 84.12$ cGy (2F-bilateral supine) and $3403.58 \pm 1482.32$ cGy (IMRT prone), $3793.84 \pm 1454.23 \mathrm{cGy}$ (IMRT prone), respectively. The maximum of mean doses obtained by right and left parotid were $1938.38 \pm 518.44 \mathrm{cGy}$ (2F-bilateral supine) and $1600.44 \pm 864.72$ cGy (2F-bilateral prone). Maximum of mean doses received by right and left temporal lobe were $3467.60 \pm$ 1696.46 cGy (2F-bilateral supine) and $3368.74 \pm 1440.76$ cGy (2F-bilateral supine), respectively.

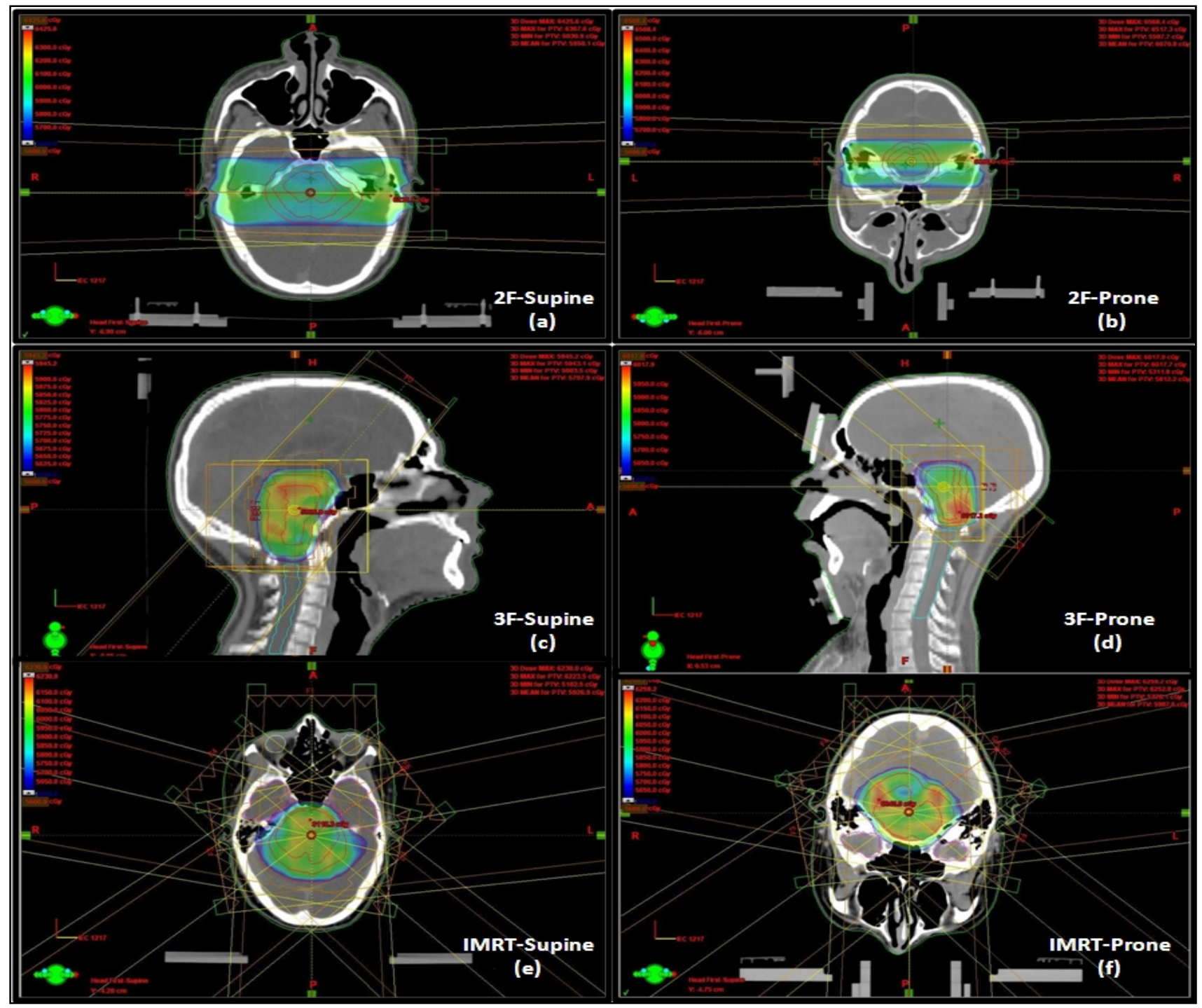

FIG.1: Beam arrangements and dose distribution of a representative patient for (a) 2-F bilateral in supine position (b) 2-F bilateral in prone position (c) 3D-CRT in supine position (d) 3D-CRT in prone position (e) IMRT in supine position (f) IMRT in prone position. 
TABLE 1: Plan evaluation indices for PTV and average of maximum and mean doses for PTV and critical organs using different treatment

\begin{tabular}{|c|c|c|c|c|c|c|}
\hline $\begin{array}{l}\text { Plan evaluation } \\
\text { indices for PTV }\end{array}$ & $\begin{array}{c}\text { 2F-bilateral } \\
\text { Supine }\end{array}$ & $\begin{array}{l}\text { 2F-bilateral } \\
\text { Prone }\end{array}$ & $\begin{array}{l}\text { 3F-3DCRT } \\
\text { Supine }\end{array}$ & $\begin{array}{l}\text { 3F-3DCRT } \\
\text { Prone }\end{array}$ & $\begin{array}{l}\text { 5F-IMRT } \\
\text { Supine }\end{array}$ & $\begin{array}{l}\text { 5F-IMRT } \\
\text { Prone }\end{array}$ \\
\hline CI PTV & $0.9525 \pm 0.03$ & $0.9558 \pm 0.02$ & $0.9636 \pm 0.03$ & $0.9668 \pm 0.02$ & $0.9824 \pm 0.01$ & $0.9759 \pm 0.01$ \\
\hline HI PTV & $1.0817 \pm 0.02$ & $1.0850 \pm 0.02$ & $1.0896 \pm 0.02$ & $1.0863 \pm 0.02$ & $1.0842 \pm 0.01$ & $1.0854 \pm 0.01$ \\
\hline $\begin{array}{c}\text { PTV \& critical } \\
\text { organ doses }(c G y)\end{array}$ & $\begin{array}{l}\text { 2F-bilateral } \\
\text { Supine }\end{array}$ & $\begin{array}{l}\text { 2F-bilateral } \\
\text { Prone }\end{array}$ & $\begin{array}{l}\text { 3F-3DCRT } \\
\text { Supine }\end{array}$ & $\begin{array}{l}\text { 3F-3DCRT } \\
\text { Prone }\end{array}$ & $\begin{array}{l}\text { 5F-IMRT } \\
\text { Supine }\end{array}$ & $\begin{array}{l}\text { 5F-IMRT } \\
\text { Prone }\end{array}$ \\
\hline PTV Dmax. & $6176.62 \pm 136.65$ & $6215.64 \pm 181.70$ & $6077.58 \pm 91.61$ & $6119.10 \pm 133.15$ & $6327.16 \pm 66.00$ & $6268.36 \pm 41.38$ \\
\hline PTV Dmean & $5883.96 \pm 104.69$ & $5896.44 \pm 107.51$ & $5873.00 \pm 69.03$ & $5855.26 \pm 45.39$ & $5960.62 \pm 44.86$ & $5949.46 \pm 42.68$ \\
\hline Chiasma Dmax. & $4988.88 \pm 513.14$ & $5078.60 \pm 371.01$ & $5441.10 \pm 557.88$ & $5455.95 \pm 228.67$ & $5153.60 \pm 450.00$ & $4861.63 \pm 507.22$ \\
\hline Rt. OPN Dmax. & $271.14 \pm 193.65$ & $237.08 \pm 148.90$ & $2402.12 \pm 784.89$ & $2109.2 \pm 886.10$ & $1997.36 \pm 1154.42$ & $1262.62 \pm 688.85$ \\
\hline Lt. OPN Dmax. & $375.66 \pm 248.89$ & $300.88 \pm 230.04$ & $2151.96 \pm 933.12$ & $2010.20 \pm 1273.01$ & $1893.10 \pm 1115.77$ & $1441.88 \pm 810.34$ \\
\hline Rt. eye Dmax. & $71.44 \pm 25.02$ & $62.74 \pm 17.95$ & $437.4 \pm 443.73$ & $789.76 \pm 555.01$ & $1441.38 \pm 818.03$ & $884.74 \pm 479.31$ \\
\hline Lt. eye Dmax. & $70.10 \pm 20.37$ & $70.36 \pm 21.30$ & $216.56 \pm 122.44$ & $503.60 \pm 383.07$ & $1211.1 \pm 717.47$ & $852.78 \pm 414.40$ \\
\hline Rt. Cochlea Dmean & $5837.02 \pm 98.20$ & $5867.74 \pm 164.28$ & $4735.74 \pm 711.17$ & $4159.74 \pm 1058.25$ & $3980.98 \pm 1239.37$ & $3403.58 \pm 1482.32$ \\
\hline Lt. Cochlea Dmean & $5882.24 \pm 84.12$ & $5658.7 \pm 490.50$ & $4859.50 \pm 753.07$ & $4373.84 \pm 1294.19$ & $3867.40 \pm 1408.52$ & $3793.84 \pm 1454.23$ \\
\hline Rt. parotid Dmean & $1938.38 \pm 518.44$ & $1718.50 \pm 355.23$ & $1156.32 \pm 131.45$ & $1131.76 \pm 257.30$ & $1472.02 \pm 290.45$ & $1218.12 \pm 466.84$ \\
\hline Lt. parotid Dmean & $1534.80 \pm 647.91$ & $1600.44 \pm 864.72$ & $1082.92 \pm 284.73$ & $1062.92 \pm 424.15$ & $1241.76 \pm 574.14$ & $1220.92 \pm 686.98$ \\
\hline Rt.T. lobe Dmean & $3467.60 \pm 1696.46$ & $3053.42 \pm 1538.46$ & $2814.66 \pm 1310.30$ & $2585.80 \pm 1155.98$ & $2727.32 \pm 1480.80$ & $2378.28 \pm 1371.25$ \\
\hline Lt.T.lobe Dmean & $3368.74 \pm 1440.76$ & $3324.86 \pm 1344.07$ & $2688.06 \pm 1204.07$ & $2376.72 \pm 1050.80$ & $2696.50 \pm 1383.24$ & $2318.60 \pm 1281.52$ \\
\hline
\end{tabular}

Abbreviations: PTV = planning target volume; $\mathrm{max}=$ maximum; $\mathrm{Lt}=$ left $\mathrm{Rt}=$ right; $\mathrm{OPN}=$ optic nerve; $\mathrm{T}=$ temporal; $\mathrm{CI}=\mathrm{cov}-$ erage index; $\mathrm{HI}=$ heterogeneity index

\section{Discussion}

As shown in Table 1, in bilateral fields (parallel opposed) planning, the PTV coverage and dose to OAR were almost similar in both supine and prone positions. Freeman et al. ${ }^{4}$ opined that standard treatment for diffuse intrinsic brain stem tumors consists of conventional radiotherapy using local fields. This treatment results in early and worthwhile improvement in neurological status, even though the overall outlook is dismal and new approaches to this tumor are clearly needed. Sparing of cochlea was not possible in bilateral field plan.

Radiation dose used in patients of brainstem glioma in available medical literature varies from 54-60 Gy in conventional fractionation. It is our institutional protocol to use $56 \mathrm{~Gy}$ in 28 fractions over 5.5 weeks in patients of brain stem glioma without radiological features of high grade malignancy. Emami et al. ${ }^{5}$ opined that one-third of brain stem can receive a dose up to $60 \mathrm{~Gy}$ to keep the 5 year complication rate below $5 \%(\mathrm{TD} 5 / 5)$.

Hua et al. ${ }^{6}$ studied the effect of cochlear dose on sensori-neural hearing loss in pediatric brain tumor patients treated with conformal radiation therapy (CRT). The authors recommended that in children receiving cranial radiotherapy to a dose of 54-59.4 Gy in 30-33 treatment fractions, cumulative dose to cochlea should be restricted below 35 Gy to minimize the risk of hearing loss. This is achievable only with highly conformal radiation therapy. 
The median survival for children with diffuse intrinsic pontineglioma (DIPG) is less than 1 year. Though we try to keep the PTV dose maximum below $107 \%$ of the prescribed dose (i.e. $59.92 \mathrm{~Gy}$ ) in bilateral parallel opposed and 3D-CRT plans, minor dose perturbations to small volume of the target are often encountered in day to day clinical practice and small excess in the permissible PTV dose maximum is accepted keeping in mind the poor prognosis in this group of patients. The PTV dose maximum in IMRT plans may exceed 107\% due to the presence of a sharp dose gradient and we use the input constraints of VPTV $107 \%<10 \%$ and VPTV $115 \%<1 \%$. Needless to say that the PTV maximum doses were higher in the IMRT plans-63.27 Gray and 62.68 Gray in supine and prone positions, respectively.

As shown in Table 1, in 3F-3D conformal plan, both supine and prone position target coverage and dose to critical structures were comparable except lower dose to cochlea in prone position plan. A modest decrease in maximum dose to optic nerves and mean dose to temporal lobes was observed for prone position plan. On the contrary, slight increase in maximum dose to eyes was noted in prone position plan. In supine position plan, superior vertex field was used in spite of the posterior location of the tumor. A posterior vertex field was not preferred in this situation to avoid beam entry through base plate and head rest (immobilization accessories), which might lead to the formation of a potential air cavity in path of the beam. In this situation, dose calculation by pencil beam algorithm in Eclipse TPS may not be accurate. Additionally, inhomogeneity correction method such as Equivalent Tissue Air Ratio (ETAR) used in pencil beam (PB) algorithm may fail to calculate the accurate dose under large inhomogeneity, as it does not consider the secondary electron transport and secondary build-up region beyond the air-cavities.

On the other hand, in prone position, beam entry for the posterior vertex field occurs from the posterior aspect of brain thereby avoiding the dose calculation uncertainty. Similarly, if the superior vertex field is placed in prone position, it will lead to attenuation through immobilization device and loss of skin sparing effect. Chiu-Tsao et al. ${ }^{7}$ has studied that how the presence of patient immobilization devices acts as a bolus during conventional and IMRT treatments and observed a significant 2D bolus effect on skin dose in the presence of patient support and immobilization devices. Frontal lobe being associated with the function of reasoning, motor skills, higher level cognition, and expressive language, it is preferable to avoid beam entry through this lobe especially in a developing brain. Occasionally prone position might not be comfortable for the patient, especially for children receiving radiation therapy under general anesthesia. In such case treatment in supine position is a good alternative. As we already discussed the limitation of pencil beam algorithm, many authors have studied the influence of dose calculation algorithms in presence of various inhomog- enities. Lu et al. mentioned that with Monte-Carlo and Acuros XB algorithm, one can expect a dose calculation accuracy approaches $100 \% .^{8}$ Amankwa-Frempong et al. ${ }^{9}$ has studied the dosimetric differences between pencil beam convolution (PBC) algorithm and anisotropic analytical algorithm (AAA) calculations in patients with lung and esophageal cancers and recommended the use of AAA algorithm in the treatment planning instead of $\mathrm{PBC}$, to avoid overestimation in dosimetry, especially for the lung tumors. Rana et al. ${ }^{10}$ reviewed the clinical dosimetric impact of the Acuros $\mathrm{XB}$ and $\mathrm{AAA}$ on real lung cancer treatment plan and observed better dose prediction accuracy of the AXB over AAA. Ojala et al. ${ }^{11}$ also opined that the Acuros $\mathrm{XB}(\mathrm{AXB})$ algorithm accuracy should be considered for reference purpose. ${ }^{11}$ In a different study, Rana et al. concluded that in comparison to the AAA, Acuros $\mathrm{XB}$ algorithm is more accurate for dose prediction in the water-equivalent material that is situated beyond the low-density medium. ${ }^{12}$

In 5F-IMRT, both the supine and prone position plans were comparable in terms of PTV coverage and homogeneity. Reduction in average maximum and mean doses to all organs at risk was observed for IMRT plan in prone position, as shown in Table 1. Good sparing of optic structures was observed in prone position compared to supine and decrease was noted for right optic nerve (dose maximum 19.97 Gy versus 12.63 Gy in supine and prone position respectively). As shown in Table 1, while comparing IMRT and 3D-CRT in both the supine and prone positions, IMRT was superior in terms of target coverage, homogeneity, and critical OAR sparing. Slight increase in dose to eye and parotid was observed in case of IMRT plan in both positions. Interestingly, dose to eye and optic nerves was lowest for 2F-bilateral plan because of absence of beam entry or exit point through these structures in both supine and prone positions. MacDonald et al. compared IMRT and 3D-CRT techniques in patients treated for high-grade glioma. The dose prescription was 59.4 Gy at 1.8 Gy per fraction using 4-10 MV photons.

The study showed that IMRT plan reduced the percent volume of brainstem receiving a dose greater than $45 \mathrm{~Gy}$ by $31 \%(p=0.004)$ and the percent volume of brain receiving a dose greater than $18 \mathrm{~Gy}, 24 \mathrm{~Gy}$ and $45 \mathrm{~Gy}$ by $10 \%(p=0.059)$, $14 \%(p=0.015)$, and $40 \%(p \leq 0.0001)$ respectively. With IMRT, the percent volume of optic chiasm receiving in excess of $45 \mathrm{~Gy}$ was also reduced by $30.40 \%(p=0.047) .{ }^{13}$ Hermanto et al. ${ }^{14}$ also compared 3D-CRT and IMRT techniques in patients of high grade glioma and concluded that with careful planning in regard to choice of beam angles, beam weighting, and recognition of potential exposure of normal tissues to exit dose, IMRT enabled improvements in target dose conformity, critical structure sparing and reduction of normal tissue integral dose. However, patients with diffuse intrinsic pontine glioma frequently present with rapid neurological deterioration and radiation therapy needs to be started expeditiously in them on the basis of clinical symptoms, signs and radiological 
features on MRI of brain (often without histological proof). Our department caters to 6000 new cancer patients annually and owing to the high patient load, the average planning time with 3D-CRT and IMRT technique is 1 day and 1 week, respectively, and this difference might be critical in this patient cohort. Moreover, the issue of higher integral dose due to larger monitor unit and beam-on time in the delivery of IMRT needs to be taken into account in the treatment of these patients, and the majority of these patients are in the pediatric age-group. Also, the issue of increased treatment time in IMRT technique needs to be reconsidered carefully in children of brainstem glioma requiring the aid of anesthesia for delivery of radiation therapy.

\section{Conclusion}

Supine and prone positions produced almost similar dose distribution in all the three techniques (2-fields bilateral, 3-fields 3DCRT, and 5-fields IMRT). At some instances, prone position plan showed better normal tissues sparing when compared to supine position. Moreover, planning in prone position is more likely to avoid attenuation due to immobilization devices and uncertainty in dose calculation under large inhomogeneity.

\section{Conflict of interest}

The authors declare that they have no conflicts of interest. The authors alone are responsible for the content and writing of the paper.

\section{References}

1. Gray A, Oliver LD, Johnston PN. The accuracy of the pencil beam convolution and anisotropic analytical algorithms in predicting the dose effects due to attenuation from immobilization devices and large air gaps. Med Phys 2009; 36:3181-91.

2. Olch AJ, Gerig L, Li H, Mihaylov I, Morgan A. Dosimetric effects caused by couch tops and immobilization devices: report of AAPM Task Group 176. Med Phys 2014; 41:061501.

3. Feuvret L, Noël G, Mazeron JJ, et al. Conformity Index: A Review. Int J Radiat Oncol Biol Phys 2006; 64:333-42.

4. Freeman CR, Farmer JP. Pediatric brain stem gliomas: a review. Int J Radiat Oncol Biol Phys 1998; 40:265-71.

5. Emami B, Lyman J, Brown A, et al. Tolerance of normal tissue to therapeutic irradiation. Int J Radiat Oncol Biol phys 1991; 21:109-22.

6. Hua C, Bass JK, Khan R, et al. Hearing loss after radiotherapy for pediatric brain tumors: effect of cochlear dose. Int J Radiat Oncol Biol Phys 2008; 72:892-99.
7. Chiu-Tsao ST, Chan MF. Evaluation of two-dimensional bolus effect of immobilization/support devices on skin doses: a radiochromic EBT film dosimetry study in phantom. Med Phys 2010; 37:3611-20.

8. Lu L. Dose calculation algorithms in external beam photon radiation therapy. Int J Cancer Ther Oncol 2013; 1:01025.

9. Amankwaa-Frempong E, Vernimmen F, Blay S, Ezhilalan R. Irradiation of lung and esophagus tumors: A comparison of dose distributions calculated by anisotropic analytical algorithm and pencil beam convolution algorithm, a retrospective dosimetric study. Int J Cancer Ther Oncol 2014; 2:020210.

10. Rana S. Clinical dosimetric impact of Acuros XB and analytical anisotropic algorithm (AAA) on real lung cancer treatment plans: review. Int J Cancer Ther Oncol 2014; 2:02019.

11. Ojala J. The acuracy of the Acuros XB algorithm in external beam radiotherapy - a comprehensive review. Int JCancer Ther Oncol 2014; 2:020417.

12. Rana S, Rogers K, Pokharel S, et al. Evaluation of Acuros XB algorithm based on RTOG0813 dosimetric criteria for SBRT lung treatment with Rapid Arc. J Appl Clin Med Phys 2014; 15:4474.

13. MacDonald SM, Ahmad S, Kachris S, et al. Intensity modulated radiation therapy versus three-dimensional conformal radiation therapy for the treatment of high grade glioma: a dosimetric comparison. J Appl Clin Med Phys 2007; 8:47-60.

14. Hermanto U, Frija EK, Lii MJ, et al. Intensity-modulated radiotherapy (IMRT) and conventional three-dimensional conformal radiotherapy for high-grade gliomas: does IMRT increase the integral dose to normal brain? Int J Radiat Oncol Biol Phys 2007; 67:1135-44. 